\title{
A Natural Language Interface for Crime-related Spatial Queries
}

\author{
Chengyang Zhang, Yan Huang, Rada Mihalcea, Hector Cuellar \\ Department of Computer Science and Engineering \\ University of North Texas \\ Denton, TX 76205 \\ Email: [chengyang,huangyan,rada]@unt.edu, hectorgcr@gmail.com
}

\begin{abstract}
Web-based mapping applications such as Google Maps or Virtual Earth have become increasingly popular. However, current map search is still keyword-based and supports a limited number of spatial predicates. In this paper, we build towards a natural language query interface to spatial databases to answer crime-related spatial queries. The system has two main advantages compared with interfaces such as Google Maps: (1) It allows query conditions to be expressed in natural language, and (2) It supports a larger number of spatial predicates, such as "within 3 miles" and "close to".

The system is evaluated using a set of crime-related queries run against a dataset that contains many spatial layers in the Denton, Texas area. The results show that our approach significantly outperforms Google Maps when processing complicated spatial queries.
\end{abstract}

\section{INTRODUCTION}

Federal and state justice departments host a large collection of heterogeneous databases, each with a complex user interface. The large number of systems and interfaces that law enforcement officers and analysts need to learn poses a serious challenge to efficient and just-in-time information access.

The ultimate goal of this line of research is to devise a robust tool that will allow law enforcement officers and analysts to interact with structured and semi-structured data using natural language. In this particular paper, we target at a subset of the problem: the development of a tool to allow automatically map crime related spatial queries expressed in natural language statements into queries understood by the underlying spatial database systems. Through this mapping tool, we target the achievement of easy-to-use interfaces that will allow access to complex databases, even for non-expert users.

A key factor motivating our project is that a natural language interaction with database systems would be clearly more "natural" and closer to human nature than the more artificial interaction performed through predefined sequences of events or statements. A form is a typical user interface that law enforcement officers and analysts use to interact with database systems. A user will fill out a form either by explicitly entering values or by choosing from values in predefined lists. The forms are static, and the expressive power is limited in most cases. Furthermore, learning how to use various forms is a very arduous task. The other way to interact with a database system is to allow users to search by keyword, but this approach lacks flexibility and expressiveness.
A flexible natural language interface to multiple database systems would result in increased information/evidence access. It reduces the training that law enforcement officers and analysts need to receive in order to access information, and allows ordinary users to interact with database systems without learning a proprietary user interface or esoteric formal language like SQL or XQuery. A natural language interaction tool is expected to be particularly useful for officers on patrol who may need ad-hoc real-time information in emergency situations.

The main contribution of this paper includes: (1) a method that allows the translation of crime-related spatial queries expressed in natural language to spatial data queries; (2) a prototype system; (3) a set of experiments that demonstrate that the system achieves results significantly better than those obtained by using Google Maps.

\section{RELATED WORK}

One of the earliest systems for creating natural language interfaces to databases relies on a pattern-matching method, using templates to map natural language sentences to semantic representations, which in turn can be mapped into database schemas [1]. The strength of this approach stands in its simplicity because it does not require any elaborate parsing or interpretation modules. On the other hand however, such simple pattern-based approaches lack scalability and sometimes can lead to serious errors of interpretation.

Slightly more sophisticated is the approach based on syntactic interpretation, in which user queries are first parsed using grammar rules, followed by a mapping between parse trees and the database query language [1], [2]. This approach has the advantage of avoiding some of the errors typically encountered in pattern-based systems because they also take into account the structure of a sentence.

More recently, the syntax-based approaches to natural language interfaces have been replaced with methods based on semantic parsing [3], [4], [5], [6]. These algorithms preserve the main idea used in the syntax-based approaches, but they also target the automatic learning of a mapping between a parse tree and the query language. The semantic parsing algorithms also seek to identify semantic classes in the input natural language statement (e.g., ship-names, rivers, etc.) in addition to syntactic dependencies. The semantic categories are used to 
enforce semantic constraints over the possible mappings and consequently improve the quality of the mapping.

Another semantic-based approach is the one used in the Precise system [7], which starts by identifying "semantically tractable questions," defined as questions likely to be correctly answered by an automatic system. The main trust is that users would like to use an automatic system only if it is guaranteed to provide a correct answer; therefore, their systems are focused on high precision, even if at the cost of lower recall.

In a related approach [8], natural language sentences are mapped to lambda-calculus encoding, which in turn is mapped to database queries. The system is trained on a manually created corpus of sentences labeled with expressions in the lambda-calculus and used to automatically induce a grammar for the domain. Moreover, the training corpus is also used to derive a lexicon related to the domain (e.g., list Utah and Texas as states). The grammar and the lexicon are then used to derive lambda-calculus expressions for new natural language statements, and consequently mapped to database queries used to locate the correct answer. The approach was found to lead to good results when evaluated on the same data sets made available by the research group at University of Texas at Austin 1 .

Finally, recently researchers have started to look into utilizing the structure of XML documents to match natural language parse trees. For instance, Nalix [9] is a prototype interactive natural language interface for querying XML. The central idea of the system is to identify a meaningful lowest common ancestor structure (called MLCAS) from the tree structure representing an XML document and use it to relate information. The system also includes an interactive component that allows the system to receive help from the users when formulating the query.

\section{Proposed Method}

We have built a prototype application for crime-related spatial queries, as a first step towards a full-fledged natural language query interface to databases. Figure 1 shows our proposed method for the prototype system. Briefly, it combines natural language processing techniques including part of speech tagging and semantic parsing with a schema matching. Instead of matching each word in the natural language input from the user, only "key words" are matched. However, unlike traditional text-based keyword matching as used in e.g., Google Maps, the "key words" identified in the query have semantic tags that are important for the extraction of spatial meanings from the database.

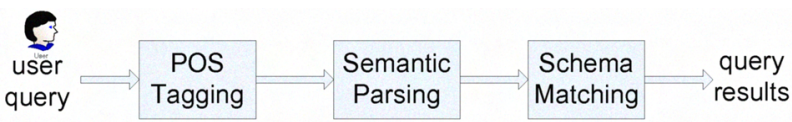

Fig. 1. Proposed method

Consider for example the following query: "I wish to find a police department within 2 miles of a law court." This query

\footnotetext{
${ }^{1} \mathrm{http} / / / \mathrm{www} . c s . u t e x a s . e d u /$ users/ml/index.cgi?page=resources.
}

is processed through the following steps in our system:

Step 1: Part-of-Speech (POS) Tagging. POS tagging is the process of determining the part of speech for each word in a sentence. In our prototype, we implemented the classic Viterbi algorithm for sequence tagging. The algorithm sweeps through all the tag possibilities for each word and computes the best sequence, according to probabilities calculated from a training data set. The key that makes this algorithm efficient is a dynamic programming framework coupled with a Markov assumption, which states that we only need to know the best sequences leading to the previous word. For training, we used a small subset of manually annotated Penn Treebank dataset. The accuracy of POS tagging in our sample test is close to $90 \%$. The result of this stage is a tagged sentence, i.e., "I/NP wish/VB to/IN find/VB a/DT police/NN department/NN within/IN 2/CD miles/NNS of/IN a/DT law/NN court/NN".

Step 2: Semantic Parsing. In this step, we try to determine three types of key words/phrases in the original query based on semantic tags generated using a syntactic parsing tree. These types are: "target object", "spatial predicate" and "reference object". "Target object" is something that the user is trying to find, for instance "police department" in our example. "Spatial predicate" is the spatial condition that is used to constrain the search, for example, "within 2 miles". The "reference object" always occur with a spatial predicate and is used as the spatial reference, for instance "law court" in the example. The "spatial predicate" and "reference object" may be omitted if a default reference location is defined in the system. In our prototype, conjunctive spatial predicates of up to size 2 are supported.

The process of semantic parsing is performed based on a set of rules derived from the syntactic parse tree. The challenge here is to figure out all the possible ways a query can be expressed using the three components. For example, a parse tree may contain following rules:

$$
\begin{array}{ll}
S \rightarrow N P & S \rightarrow N P V P \\
S \rightarrow V P & V P \rightarrow \text { Verb } N P \\
N P \rightarrow \text { Adj } N P P P & N P \rightarrow \text { Noun } \\
P P \rightarrow \text { Prep NP } & P P \rightarrow \text { Prep } C D N P P P
\end{array}
$$

Based on these rules we may determine that the head word of the first "NP" after the verb phrase is the "target object", or the first "NP" itself is the "target object" if no verb phrase can be found in the sentence. Similarly, we may use the position of the preposition phrase to determine the reference object and the spatial predicate. The result of this stage is a triplet that contains the three semantic predicates, i.e., ("police department", "within 2 miles", "law court").

Step 3: Schema Matching. At this step we try to find the target and reference spatial objects from the backend spatial database, and then perform a spatial join to test whether the spatial predicate is satisfied. A result is returned if the predicate is true. The process of finding matching objects is performed using a distance function that determines the most similar words in all the tables, based on the table schema names and on the actual content of the database tables. The challenge here is to appropriately determine the weighted distance function when multiple matches occur. In our example, 
we are looking for "police department" and "law court." When we find tuples that are relevant, we retrieve their spatial extent, and determine whether they are within 2 miles of each other. The final result is converted to Keywhole Markup Language (KML) format for display on a map interface.

\section{Prototype System Evaluation}

Our prototype system includes a backend spatial database (PostgreSQL), a logic layer implemented using Perl scripts, and a web-based query interface implemented using Php. A screenshot of the query interface and the result displayed for the sample query is shown in Figure 2.

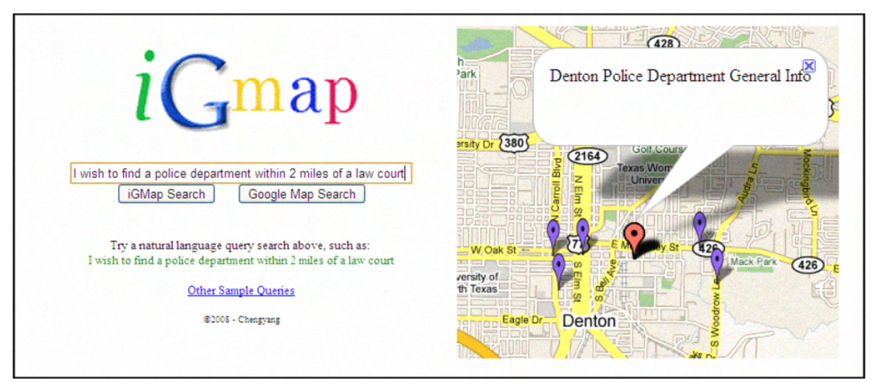

Fig. 2. Query Interface

The spatial dataset used in our system is obtained from the Denton County, Texas. It contains 32 tables (layers) for various locations in the Denton area, including crime-related places such as police departments and courts. Most of these objects can be found on Google Maps as well. Therefore our dataset can be considered as a small subset of the Google Maps backend data.

For evaluation purposes, we collected 30 different queries related to crime, with human prepared answers. We list below five sample queries from this data set:

- "What are all the fire stations within 5 miles from a police department?"

- "Find crime stoppers near a school district."

- "Find a criminal attorney close to the Golden Triangle Mall."

- "Find a law office no further than 5 miles from a law court."

- "Find an emergency room less than 5 miles from Stemmons Freeway."

We issue queries to both our system and to Google Maps. We then compare the precision and the recall value for the returned answers. Since Google Maps sometimes gives a large list of answers, while our dataset is relatively small, we only use the top 10 answers from both our system and from Google Maps. Moreover, for a fair comparison, we always add the constraint "near Denton, TX" to the Google Maps query.

The results are shown in Table I. As seen in the table, our system can achieve precision of nearly $60 \%$, which is significantly higher than that of Google Maps (3.3\%). This indicates that without understanding the spatial information in the natural query condition, it is difficult to accurately locate the relevant information based solely on keyword matching.
Our experiments also show that the recall of Google Maps is four times lower than our system; this is true even when we consider the top 20 or more returned results.

TABLE I

SySTEM EVALUATION RESULT

\begin{tabular}{llll}
\hline System & Precision(\%) & Recall(\%) & F-Measure(\%) \\
\hline \hline Our system & 59.7 & 31.9 & 41.6 \\
Google Maps & 3.3 & 6.9 & 4.5 \\
\hline
\end{tabular}

\section{CONCLUSION AND FUTURE WORK}

In this paper, we proposed a method for building a natural language query interface to spatial database systems. We implemented a prototype system for crime-related application and demonstrated its effectiveness compared to the widelyused Google Maps. The experimental results show that an approach that relies on natural language processing techniques applied on the user input can be practically and effectively used.

In our future work, we plan to extend our system by increasing the dataset size, and improving the accuracy of the tagging and parsing algorithms. We will collect more user queries and improve the system performance based on a larger evaluation dataset. We also plan to build a more flexible interface that will allow the results to be easily exported into KML files.

\section{REFERENCES}

[1] I. Androutsopoulos, G. Ritchie, and P. Thanisch, "Natural language interfaces to databases - an introduction," Journal of Natural Language Engineering, vol. 1, no. 1, 1995.

[2] W. Woods, R. Kaplan, and B. Webber, "The Lunar sciences natural language information system," Bolt Beranek and Newmann, Tech. Rep., 1972.

[3] R. Ge and R. J. Mooney, "A statistical semantic parser that integrates syntax and semantics," in Proceedings of the Ninth Conference on Computational Natural Language Learning (CoNLL-2005), Ann Arbor, MI, Jul. 2005, pp. 9-16.

[4] R. J. Kate and R. J. Mooney, "Using string-kernels for learning semantic parsers," in Proceedings of the 21st International Conference on Computational Linguistics and 44th Annual Meeting of the Association for Computational Linguistics (COLING/ACL-06), Sydney, Australia, July 2006, pp. 913-920.

[5] R. J. Mooney, "Learning for semantic parsing," in Computational Linguistics and Intelligent Text Processing: Proceedings of the 8th International Conference, CICLing 2007, Mexico City, A. Gelbukh, Ed. Berlin: Springer Verlag, 2007, pp. 311-324.

[6] Y. Wong and R. J. Mooney, "Learning for semantic parsing with statistical machine translation," in Proceedings of Human Language Technology Conference / North American Chapter of the Association for Computational Linguistics Annual Meeting (HLT-NAACL-06), New York City, NY, 2006, pp. 439-446.

[7] A. Popescu, A. Armanasu, and O. Etzioni, "Modern natural language interfaces to databases: Composing statistical parsing with semantic tractability," in Proceedings of the 20st International Conference on Computational Linguistics (COLING 2004), Geneva, Switzerland, 2004.

[8] L. Zettlemoyer and M. Collins, "Learning to map sentences to logical form: Structured classification with probabilistic categorial grammars," in Proceedings of the Twenty First Conference on Uncertainty in Artificial Intelligence (UAI-05), 2005.

[9] Y. Li, H. Yang, and H. Jagadish, "NaLIX: an interactive natural language interface for querying XML," in Proceedings of SIGMOD 2005, Baltimore, MD, 2005. 